\title{
Simple Measurement System for Biological Signal Using a Smartphone
}

\author{
Ryoichi Miyauchi $^{1}$, Koichi Tanno ${ }^{2}$, Hiroki Tamura ${ }^{3}$ \\ ${ }^{1}$ Interdisciplinary Graduate School of Agriculture and Engineering, University of Miyazaki, Japan \\ ${ }^{2}$ Department of Electrical and Systems Engineering, University of Miyazaki, Japan \\ ${ }^{3}$ Department of Environmental Robotics, University of Miyazaki, Japan
}

\section{Article Info}

Article history:

Received Mar 20, 2018

Revised Jun 12, 2018

Accepted Jun 30, 2018

\section{Keyword:}

AC/DC converter

Biological signal

Signal conditioning

Smartphone

\begin{abstract}
This paper describes simple measurement system for biological signal using smartphone. The proposed system consists of an instrumentation amplifier, a filter and an AC/DC converter. The biological signal is converted to the digital data through the microphone terminal with $\mathrm{A} / \mathrm{D}$ converter in the smartphone. In many cases, the circuits require the power sources such as the cell batteries, however, the proposed system is supplied the power through the earphone terminal of the smartphone. Therefore, the proposed system no require the batteries. The software of this system parallelizes the processing so that the earphone output and the microphone terminal can be executed at the same time. The proposed system was verified through the measurement of surface electromyogram using discrete parts and iOS. Results of experimentation, the proposed system was operating correctly.
\end{abstract}

Copyright $(0) 2018$ Institute of Advanced Engineering and Science. All rights reserved.

Corresponding Author:

Koichi Tanno,

Department of Electrical and Systems Engineering,

Institute of Education and Research for Engineering, University of Miyazaki,

1-1 Gakuenkibanadai-Nishi, Miyazaki 889-2192, Japan.

Email: tanno@cc.miyazaki-u.ac.jp

\section{INTRODUCTION}

In recent years, improving the quality of life and increasing healthspan is become global concern and challenges [1]-[5]. For this reason, management of health on a daily basis is very important, and measuring the own vital data, such as heartbeats, pulses etc, using various equipment are essential. On the other hands, wearable devices, which evolves at a rapid pace, are one of the equipment to measure the biological signal. In the future, own health management would become almost equal to management by personal trainers by analyzing measured data using AI and deep learning [6], [7]. However, it is difficult to realize the above system because the system requires the many functions and the cost will become high. Therefore popularization of the wearable devices is delayed.

On the other hands, biological signals, such as Electromyogram (EMG), Electrooculogram (EOG), Electroencephalogram (EEG), Electrocardiogram (ECG), are well known as the vital data. In general, biological signals have characteristics of small amplitude ( $\square \mathrm{V}$ to $\mathrm{mV}$ ) and low frequency range (DC to few $\mathrm{KHz}$ ). In order to implement the biological signal measurement system, not only an analog circuit such as the amplifier, filter, Analog to Digital (A/D) converter, but also a digital circuit such as processor and memory are required. In addition, the general wearable devices are consists of a measurement terminal, a display terminal, and a wireless communication block, etc. That is to say, these kind of systems become complex and high cost.

In this research, in order to realize the system simply and reduce the cost, a new architecture for biological measurement system using a smartphone is proposed. The proposed system consists of an instrumentation amplifier, a filter and an AC/DC converter and these circuits are supplied the power through 
the earphone terminal of the smartphone. Therefore, the proposed system no require the batteries. The proposed system was realized by using discrete parts and verified through the measurement of surface EMG (s-EMG). The experimental results are shown in this paper.

\section{CONVENTIONAL MEASUREMENT SYSTEM}

Figure 1 shows example of the stationary type measurement system. The stationary type measurement system is constructed by analog front end (AFE), and PC. In addition, AFE and PC are connected by USB, therefore, this system is one of the wired system. AFE consists of an amplifier, a filter and an A/D converter.

The stationary type measurement system is often used for medical, and this system has some advantages; this system has high accuracy and multi input channels and can be monitored measured data on real time. On the other hands, this system has an disadvantage of low portability.

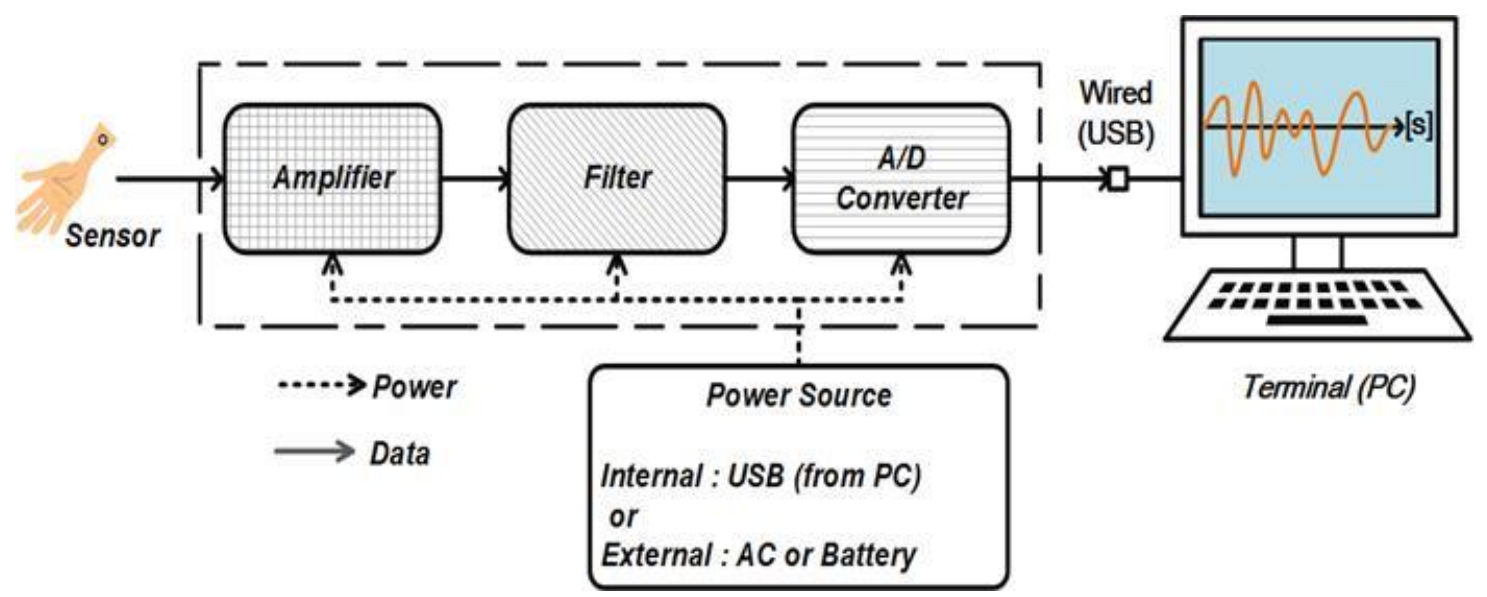

Figure 1. Stationary type measurement system

Figure 2 shows example of the wearable type measurement system. The wearable type measurement system consists of AFE, PC, memory, communicator and battery. And this system is one of the wireless measurement system. The advantage of the wearable type measurement system is high portability, however, it requires many function and high cost.

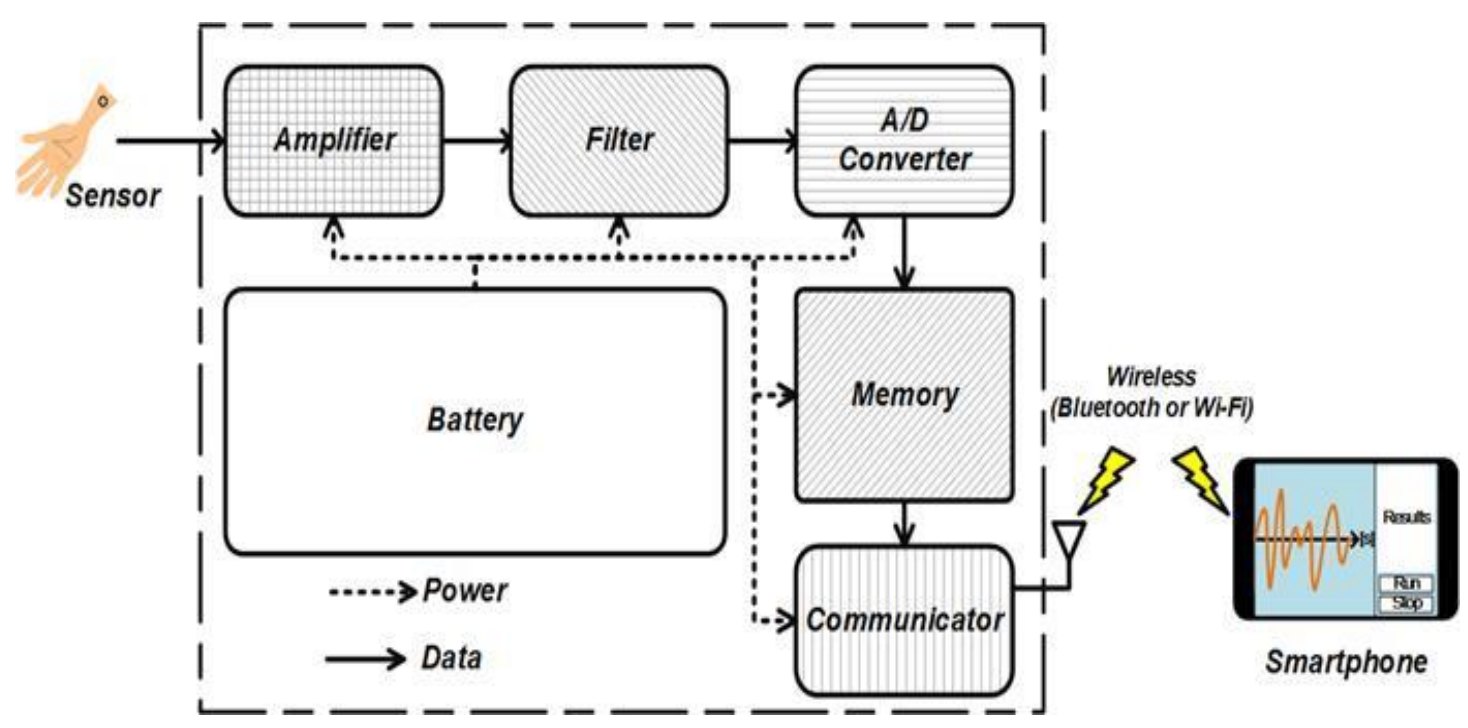

Figure 2. Wearable type measurement system 


\section{PROPOSED MEASUREMENT SYSTEM}

\subsection{System Architecture}

Figure 3 is proposed measurement system, and we call this system Signal Conditioner. The proposed system consists of three circuit blocks; the instrumentation amplifier (IA), the filter and AC/DC converter. The IA is amplified input signal from sensor because the amplitude of biological signals very small. In this system, we adopted the IA, which has very high input impedance and CMRR. Furthermore, gain of the IA can be choose by the selecting a resistor. The low pass filter (LPF) is adopted in this system for eliminating the noise other than the target signal. Because the frequency of the biological signal is relatively low in general, the cutoff frequency of the LPF is less than $1000 \mathrm{~Hz}$ in this system. The AC/DC converter of the last block is used for generating the DC power supplies. The audio signals, which are the sinusoidal waves, are generated by the application program in the smartphone, and the generated audio signals are outputted through earphone terminal of the smartphone. And then, these audio (sinusoidal) signals are converted to DC by the AC/DC converter. This DC signals are used for the power sources for operating the circuits in the proposed system.

Comparing the conventional stationary type measurement system shown in Figure 1, the proposed system can remove the A/D converter because the smartphone can receive analog signal directly through the microphone terminal, and the A/D converter is implemented in the smartphone. In addition, comparing the conventional wearable type measurement system, the proposed system can remove the A/D converter, the communicator and the battery.

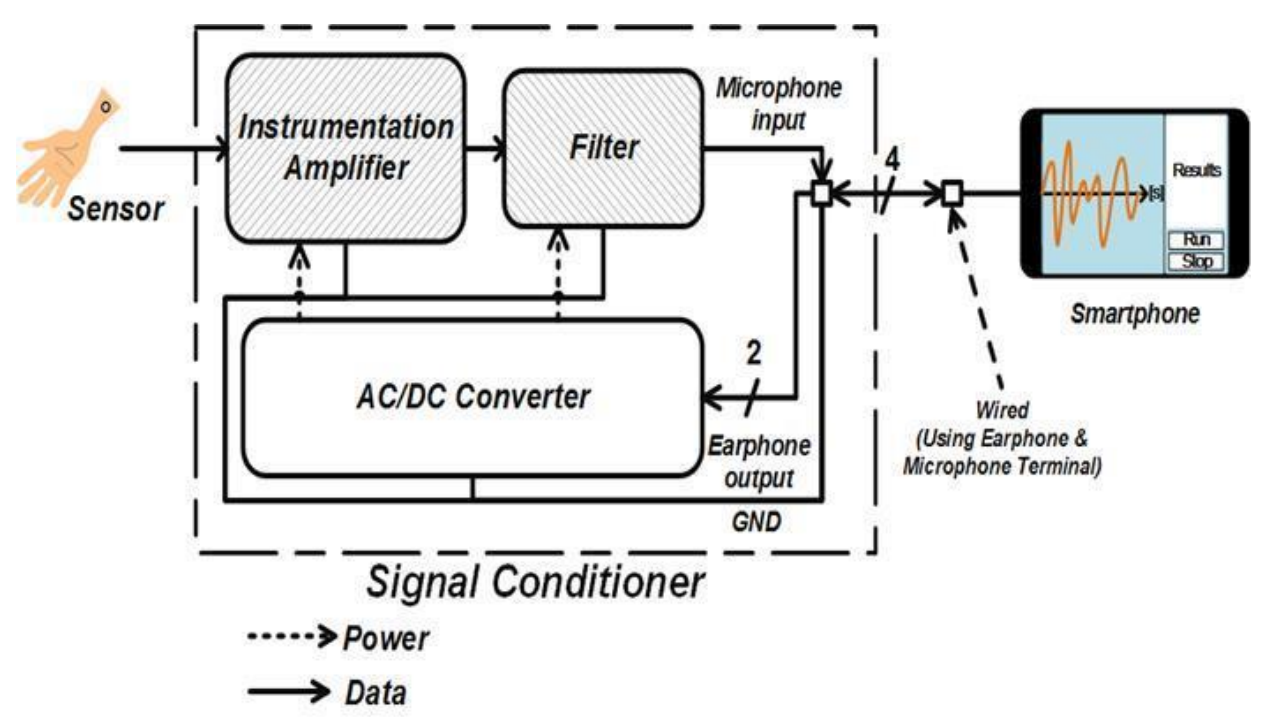

Figure 3. Proposed measurement system

\subsection{System Realization}

Figure 4 shows the system realization using discrete parts. $R_{G}$ is resistor for selecting the gain of IA which is given by equation 1 . In equation $1 \square$ is constant value which depends on the device (decided by register network in IA), and selecting small $R_{G}$ can be obtained high gain if we need.

$$
\operatorname{gain}=1+\frac{\alpha}{R_{G}}
$$

$R_{F 1}$ and $C_{F 1}$ constitute of the LPF, and this LPF is necessary for reducing the noise in this system. The main noise in this system is the ripple which is occurred from AC/DC converter, and the frequency of this noise is more than a few $\mathrm{kHz}$. In the actual realization, we adopted a first order LPF with the cut-off frequency is approximately $600 \mathrm{~Hz}$.

AC/DC converter shown in Figure 4 is the sinusoidal wave to DC converter with voltage doubler, which outputs both positive and negative voltages. In the biological signal measurement system, analog circuit blocks need positive and negative voltage sources because the bias voltages is not added to the input signal from sensor. Furthermore, the output voltage from the earphone terminal is not generally so high, therefore we employed the AC/DC converter with voltage doubler in order to operate all circuit blocks. 
$D_{1}$ and $C_{2}$ constitute the well-known half-wave rectifier circuit [8][9]. The AC/DC converter with voltage doubler can be realized by adding to $D_{2}$ and $C_{l}$. By adding $D_{2}$ and $C_{1}$, the negative half cycle of the input signal from the earphone terminal charge $C_{l}$ and the next positive half cycle charge $C_{2}$ with adding the voltage of $C_{l}$. As the results, the positive voltage doubler can be achieved. In the same way, the circuit of the bottom side in AC/DC converter shown in Figure 4 also operates as the negative voltage doubler because the circuit has complementary structure of upper side one.

Figure 5 shows activity diagram of proposed system. This system has to execute input and output at the same time. In addition, input program and output program always have to work continuously. Therefore, that is realized using multi-thread programming. And, some button is required for user interface(UI).

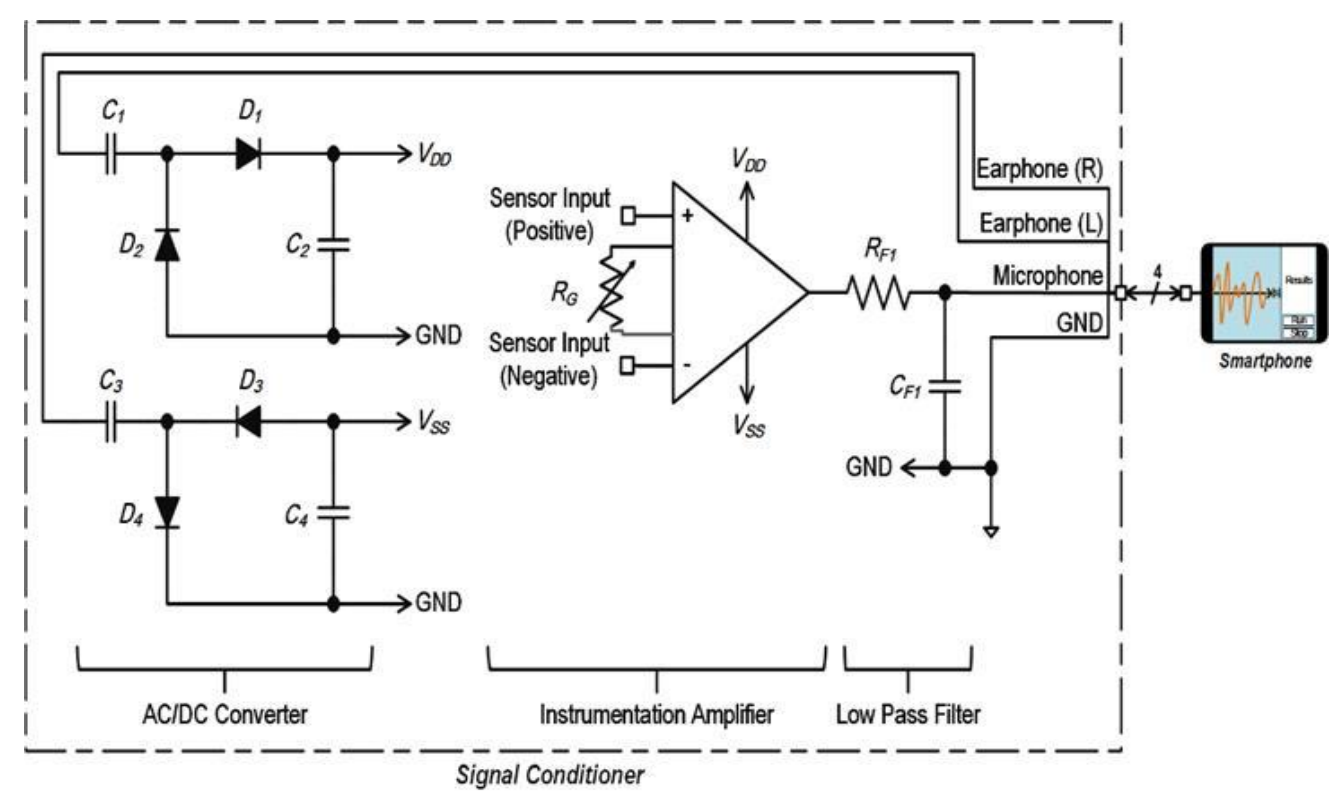

Figure 4. System realization

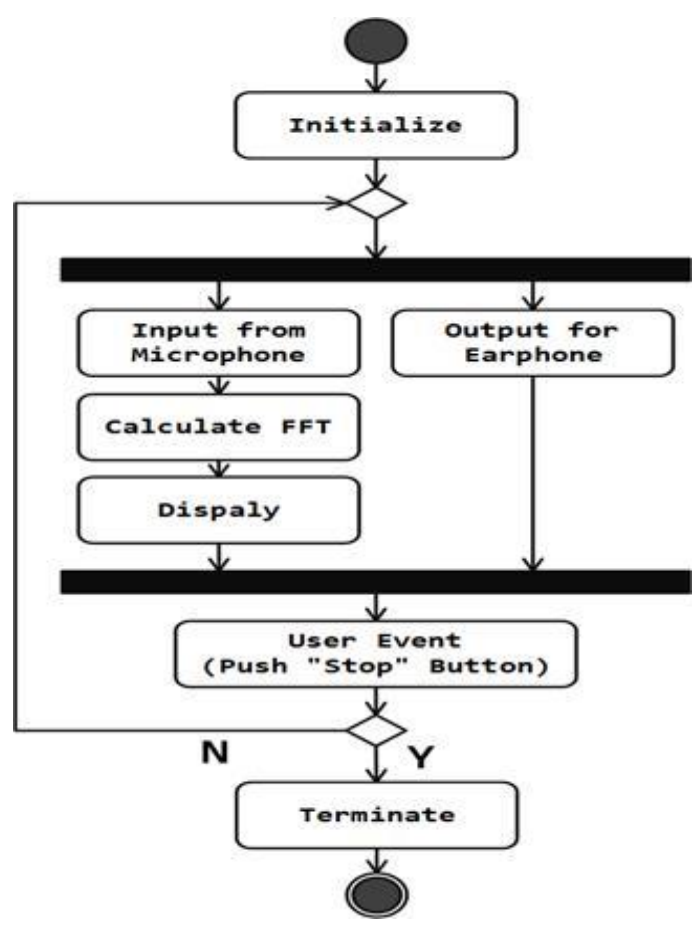

Figure 5. Activity diagram of proposed systemEXPERIMENTAL AND COMPARISON RESULTS 
Figure 6 and Table 1 show photograph of overall of the proposed system and experimental condition, respectively. From Figure 6, it is clear that smartphone and the signal conditioner are connected only one wire. Gain of IA and cut-off frequency of LPF are configured 51 times and $605 \mathrm{~Hz}$, respectively. And, the output signals of the earphone terminal consists of the amplitude of $1.5 \mathrm{~V}$ with the frequency of $10 \mathrm{kHz}$.

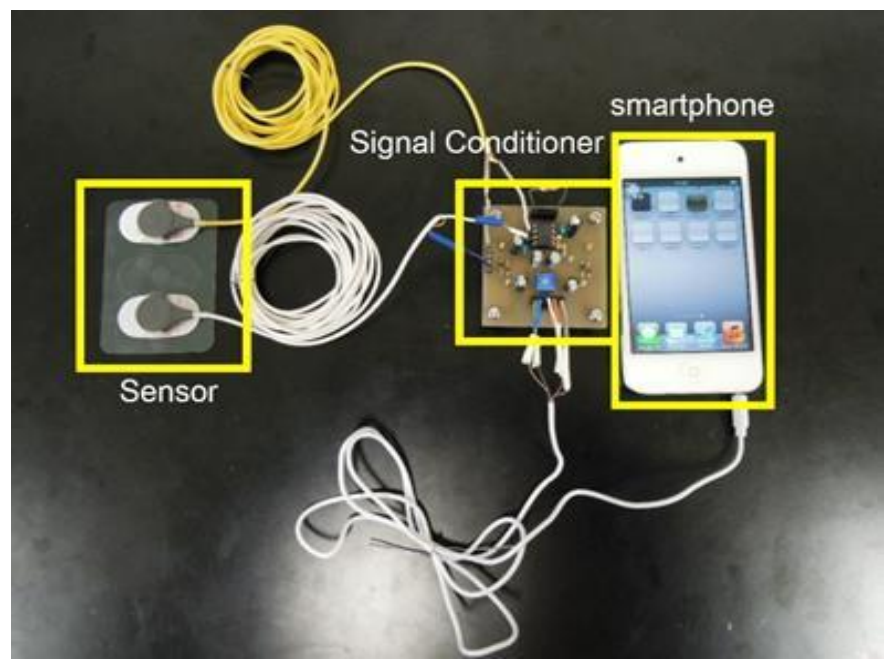

Figure 6. Photograph of overall of the proposed system

Table 1. Experiment Condition

\begin{tabular}{ll}
\hline \multicolumn{1}{c}{ Item } & \multicolumn{1}{c}{ Value } \\
\hline Smartphone & Ipod touch 4th Gen. \\
Instrumentation Amplifier & INA118 [10] \\
$D_{1}-D_{4}$ & $1 \mathrm{~N} 4148[11]$ \\
$C_{I}-C_{4}$ & $4.7 \mu \mathrm{F}$ \\
$R_{G}$ & $1 \mathrm{k} \Omega$ \\
$R_{F 1}$ & $560 \Omega$ \\
$C_{F 1}$ & $0.47 \mu \mathrm{F}$ \\
\hline
\end{tabular}

In this experiments, we employed the s-EMG signal as the biological signal. Figure 7 and Table 2 show results of s-EMG measurement. In Figure 7, waveform, which are viewed on the display of the smartphone, shows results of the FFT analysis for the measured s-EMG signal. From Figure 7, we can confirm that the waveforms in the case of neutral, pronation and grip are different. Furthermore, we could confirm that FFT results were changed according as moving the arm. And, Table 2 shows experimental result for each examinee, and it is indicated that the proposed system could use generally. From these experimental results, the proposed system can be operated correctly.

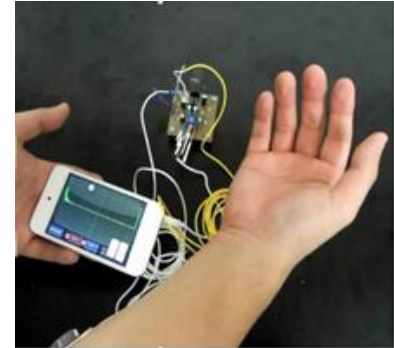

(a) Neutral

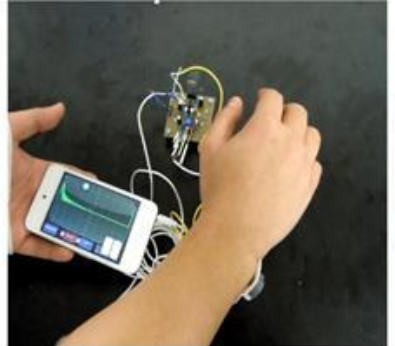

(b) Pronation

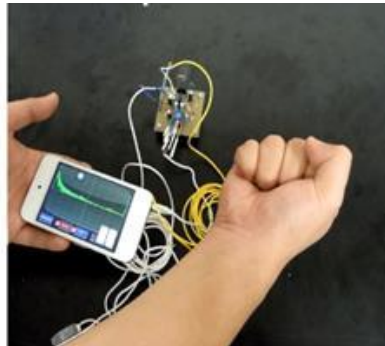

(c) Grip

Figure 7. Photograph of overall of the proposed system

Table 2. Experimental Result for Each Examinee Examinee Right Arm Left Arm 


\begin{tabular}{lll}
\hline A & 0 & 0 \\
B & $\circ$ & 0 \\
C & $\circ$ & 0 \\
D & $\circ$ & 0 \\
E & $\circ$ & 0 \\
\hline
\end{tabular}

Table 3 shows comparison result between the proposed and conventional systems. From Table 3, the proposed system could realize battery-less and low costs. However, comparing the wearable type measurement system, portability is decreasing by using wired connection. And comparing the stationary type measurement system the number of channels is only one in this situation. However, this disadvantage will be able to overcome the signal multiplexing techniques, which were proposed in the past [12], [13].

Table 3. Comparison Result

\begin{tabular}{cccc}
\hline Item & Proposed & Stationary & Wearable \\
\hline Connection type & wired & wired & wireless \\
Battery & not require & not require & require \\
Costs (Number of Item) & Low & High & High \\
Portability & Mid. & Low & High \\
Resolution & depends on smartphone & High & Mid. \\
Number of channels & $1^{*}$ & multi & 1 \\
\hline
\end{tabular}

* The number of channels can expand by using the signal multiplexing techniques

\section{CONCLUSION AND FUTURE WORKS}

In this paper, very simple and battery-less biological measurement system, which consists of IA, filter and AC/DC converter, has been proposed. We could confirmed that the proposed measurement system operated correctly through the experiment with implemented system using the discrete parts and the smartphone. Comparing the conventional systems, the proposed system has advantages which are battery-less and low costs. In summary, the proposed system can be achieved the advantages by using implemented functions of the smartphone positively. The future works are as follows; 1) expanding the number of channels, 2) implementing the integrated circuit of the signal conditioner by using references [14]-[16], and 3 ) comparing and evaluating the proposed system with marketed products.

\section{REFERENCES}

[1] United Nations, "Population Division (2017)," World Population Prospects: The 2017 Revision, Working Paper No.ESA/P/WP/248.

[2] D. Madhavi, B.V. Ramana, "De-Identified Personal Health Care System Using Hadoop", IAES International Journal of Electrical and Computer Engineering, vol. 5, no. 6, Dec. 2015.

[3] T. Prayoga, J. Abraham, "Behavioral Intention to Use IoT Health Device: The Role of Perceived Usefulness, Facilitated Appropriation, Big Five Personality Traits, and Cultural Value Orientations", IAES International Journal of Electrical and Computer Engineering, vol. 6, no. 4, Aug. 2016.

[4] J.W. Lee, et al., "User Analysis Mechanisms based Mobile Fitness System", IAES International Journal of Electrical and Computer Engineering, vol. 6, no. 6, Dec. 2016.

[5] A. Setiabudi, et al., "CMOS Temperature Sensor with Programmable Temperature Range for Biomedical Applications", IAES International Journal of Electrical and Computer Engineering, vol. 8, no. 2, April 2018.

[6] A. Kazantsev, et al., "Development of e-health network for in-home pregnancy surveillance based on artificial intelligence", 2012 IEEE-EMBS International Conference on Biomedical and Health Informatics, pp. 82-84, 2012.

[7] Z. Che, Y. Liu, "Deep Learning Solutions to Computational Phenotyping in Health Care", 2017 IEEE International Conference on Data Mining Workshops, pp. 1100-1109, 2017.

[8] T. Lehmann, Y. Moghe, "On-Chip Active Power Rectifiers for Biomedical Applications," IEEE International Symposium on Circuits and Systems, Vol.1, pp. 524-527, 2005.

[9] D. Karolak, et al., "Design Comparison of Low-Power Rectifiers Dedicated to RF Energy Harvesting," IEEE International Conference on Electronics, Circuits, and Systems, pp. 524-527, 2012.

[10] INA118, Precision, Low Power Instrumentation Amplifier, Texas Instruments Inc., Dallas, Sep. 2000[Online], available from http://www.ti.com/product/ina118, accessed on March 5, 2018.

[11] 1N4148, High Conductance Fast Diode, On Semiconductor Inc., Phoenix, June 2015[Online], available from http://www.onsemi.com/PowerSolutions/product.do?id=1N4148, accessed on March 5, 2018.

[12] K. R. Moghaddam, et al., "A 24-channel implantable neural recording based on time and frequency-division multiplexing", Analog Integrated Circuits and Signal Processing, Vol. 79, Issue 3, pp. 493-506, June, 2014.

[13] X. Zhao, et al., "Analog Signal Compression and Multiplexing Techniques for Healthcare Internet of Things", IEEE International Conference on Mobile Ad Hoc and Sensor Systems, pp. 398-406, 2017. 
[14] Y. Rao, D.P. Arnold, "An AC/DC Voltage Doubler with Configurable Power Supply Schemes for Vibrational Energy Harvesting", IEEE Applied Power Electronics Conference and Exposition, pp. 2844-2851, 2013.

[15] Z. Abidin, et al., "Low Common-Mode Gain Instrumentation Amplifier Architecture Insensitive to Resistor Mismatches", IAES International Journal of Electrical and Computer Engineering, vol.6, no. 6, Dec. 2016.

[16] Z. Abidin, et al., "A New Instrumentation Amplifier Architecture Based on Differential Difference Amplifier for Biological Signal Processing", IAES International Journal of Electrical and Computer Engineering, vol.7, no. 2, April 2017.

\section{BIOGRAPHIES OF AUTHORS}
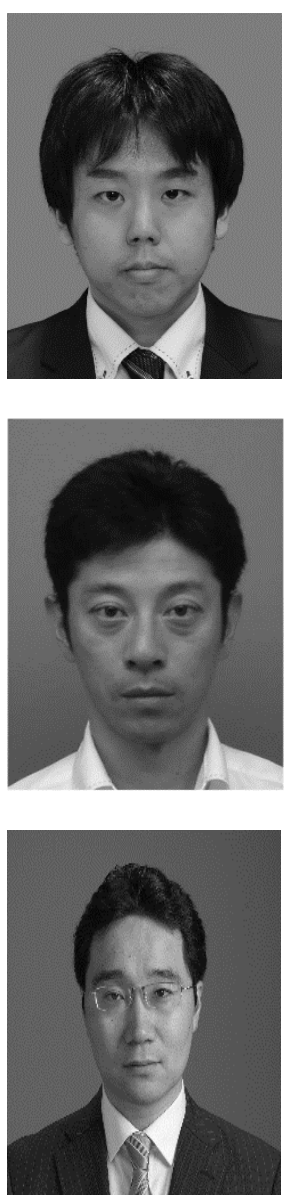

was born in Kagoshima, Japan, on March 3rd, 1986. He received B. E. and M. E. degrees from Faculty of Engineering, University of Miyazaki, Miyazaki, Japan, in 2008 and 2010, respectively. He is currently studying to get Dr. Eng. degree at University of Miyazaki. From 2010 to 2015, he joined the Hitachi ULSI Systems Co., Ltd., Tachikawa, Japan. He was engaged in development of application software for Windows and development of voice recognition system for car navigation system. His research interests are biological signal measurement system and analog integrated circuit.

was born in Miyazaki, Japan, on April 22, 1967. He received B. E. and M. E. degrees from the Faculty of Engineering, University of Miyazaki, Miyazaki, Japan, in 1990 and 1992, respectively, and Dr. Eng. degree from Graduate School of Science and Technology, Kumamoto University, Kumamoto, Japan, in 1999. From 1992 to 1993, he joined the Microelectronics Products Development Laboratory, Hitachi, Ltd., Yokohama, Japan. He was engaged in research on low-voltage and low-power equalizer for read channel LSI of hard disk drives. In 1994, he joined University of Miyazaki, where he is currently a Professor in the Department of Electrical and Systems Engineering. His main research interests are in analog integrated circuit design and multiple-valued logic circuit design. Dr. Tanno is a member of IEEE.

received the B. E. and M. E. degree from Miyazaki University in 1998 and 2000, respectively. From 2000 to 2001, He was an Engineer in Asahi Kasei Corporation, Japan. In 2001, He joined Toyama University, Toyama, Japan, where He was a Technical Official in Department of Intellectual Information Systems. In 2006, He joined Miyazaki University, Miyazaki, Japan, where He was an Assistant Professor in Department of Electrical and Electronic Engineering. In 2012, He is currently a Professor in the Department of Environmental Robotics. His main research interests are Neural Networks and Optimization Problems. In recent years, He has the interest in Biomedical Signal Processing using Soft Computing. 